\title{
Positioning Accuracy of Single Frequncy GNSS PPP by CLAS Comparing with MADOCA Products
}

\author{
Dai Mimura, Katsumasa Miyatake*, Yukihiro Kubo, and Sueo Sugimoto \\ Department of Electrical and Electronic Engineering, Ritsumeikan University \\ Noji-Higashi, Kusatsu City, Shiga 525-8577 Japan \\ * Mechatronics Department, Advanced Technology R\&D Center, Mitsubishi Electric Corporation, \\ Amagasaki City, Hyogo 661-8661, Japan
}

\begin{abstract}
In this paper, we present the single frequency PPP algorithms based on our GR models with applying CLAS(Centimeter Level Augmentation Service) data from Japan Quasi-Zenith-Satellite-System (QZSS), comparing with the positioning results applying the MADOCA (MultiGNSS Advanced Demonstration tool for Orbit and Clock Analysis) Products which had been developed by JAXA (Japan Aerospace Exploration Agency) and are provided by GPAS (Global Positioning Augumentation Service Corporation), Throughout the numerical experiments the total 3DMMS error by applying CLAS is approximately 0.28 [m], in contrary with the total 3DMMS error by applying MADOCA Productsis being aproximately 0.52 [m].
\end{abstract}

\section{Introduction}

In this paper, we implement the satellite orbits and clocks correction data from the CLAS [1] or MADOCA Products [2]-[3] into our PPP algorithms. Additionally, Ionosphere delay is obtained from CLAS or GIM. and Tropospheric delay is obtained from CLAS or Saastamoinen model.

First of all, we review the previous PPP algorithms based on Global navigation satellite system Regression models (referred as GR models) which have been developed in [4]-[5]. By presenting GR equations according to [4]-[5], we derive single frequency PPP algorithms. Then, we shows the experimental results of positioning accuracy of using CLAS with compering MADOCA Products.

\section{Measurement Model}

Similarly to [5], we only describe the case of L1 frequncy GNSS Regression (GR) models for the observed positioning data consisting of $\mathrm{L} 1$ carrier phases and pseudoranges based on C/A code due to simpler description and the commercial usage of positioning. Namely, we consider the following fundamental measurements of L1 band carrier phases $\Phi_{L 1, u}^{p}(t)$ and the pseudoranges $\rho_{C A, u}^{p}(t)$ based on $\mathrm{C} / \mathrm{A}$ codes, respectively, as follows:

$$
\begin{aligned}
\rho_{C A, u}^{p}(t)= & r_{u}^{p}\left(t, t-\tau_{u}^{p}\right)+c\left[\delta t_{u}(t)-\delta t^{p}\left(t-\tau_{u}^{p}\right)\right] \\
& +\delta I_{u}^{p}(t)+\delta T_{u}^{p}(t)+\delta b_{C A}^{p}+d_{r e l}^{p} \\
& +\Delta p_{a n t}+e_{C A, u}^{p}(t), \\
\Phi_{L 1, u}^{p}(t)= & \lambda_{1} \varphi_{L 1, u}^{p}(t) \\
=\quad & r_{u}^{p}\left(t, t-\tau_{u}^{p}\right)+c\left[\delta t_{u}(t)-\delta t^{p}\left(t-\tau_{u}^{p}\right)\right] \\
& -\delta I_{u}^{p}(t)+\delta T_{u}^{p}(t)+\delta b_{L 1}^{p}+d_{r e l}^{p} \\
& +\Delta p_{a n t}+\lambda_{1}\left(N_{L 1, u}^{p}+\Delta \phi_{L 1, u}^{p}\right) \\
& +\lambda_{1} \varepsilon_{L 1, u}^{p}(t) .
\end{aligned}
$$

In (1)-(2), $r_{u}^{p}\left(t, t-\tau_{u}^{p}\right)$ is the geometric distance between the receiver $u$ at the time $t$ and the satellite $p$ at the time $t-\tau_{u}^{p}\left(\tau_{u}^{p}\right.$ denotes the travel time from the satellite $p(p=$ $\left.1, \ldots, n_{s}\right)$ to the receiver $\left.u\right)$. Namely, $r_{u}^{p}(t) \equiv r_{u}^{p}(t, t-$ $\left.\tau_{u}^{p}\right)=\left\|u(t)-s^{p}\left(t-\tau_{u}^{p}\right)\right\|$, where $u \equiv\left[x_{u}, y_{u}, z_{u}\right]^{\mathrm{T}}$ and $s^{p} \equiv\left[x^{p}, y^{p}, z^{p}\right]^{\mathrm{T}}$ are the user (unknown) and satellite positions, respectively. Also, $c\left(\approx 2.99792458 \times 10^{8}[\mathrm{~m}]\right)$ denotes the speed of light, $\left(\delta t_{u}\right)$ and $\left(\delta t^{p}\right)$ are the receiver and satellite clock errors respectively, $\left(\delta I_{u}^{p}\right)$ is the Ionosphere delay. $\left(\delta T_{u}^{p}\right)$ is the Tropospheric delay, $f_{1}(=1575.42[\mathrm{MHz}])$ and $\lambda_{1}$ are the central frequency and the wave length of the $L 1$ carrier wave, $\left(\delta b_{C A}^{p}\right)$ and $\left(\delta b_{L 1}^{p}\right)$ are the code bias and the phase bias respectively for the satellite $p .\left(d_{r e l}^{p}\right)$ is relativistic delay $[\mathrm{m}] .\left(\Delta p_{a n t}\right)$ is antenna phase center offsets (PCO) and antenna phase center variation $(\mathrm{PCV})[\mathrm{m}] .\left(N_{L 1, u}^{p}\right)$ denotes integer ambiguity between the satellite $p$ and the receiver $u,\left(\Delta \phi_{L 1, u}^{p}\right)$ is the phase wind up correction and $e$ and $\varepsilon$ denote measurement errors.

Then, we take the 1st order Taylor series approximation around the previous estimated value $u=\hat{u}$ and $s^{p}=\hat{s}^{p}$ such that we have the GR equations [5]:

$$
\begin{aligned}
\rho_{C A, u}^{p} \cong \quad & g_{\hat{u}}^{\hat{p}}\left(u-s^{p}\right) \\
& +c\left(\delta t_{u}-\delta t^{p}\right) \\
& +\delta I_{u}^{p}(t)+\delta T_{u}^{p}(t)+\delta b_{C A}^{p} \\
& +d_{r e l}^{p}+\Delta p_{\text {ant }}+e_{C A, u}^{p}(t), \\
\Phi_{L 1, u}^{p} \cong \quad & g_{\hat{u}}^{\hat{p}}\left(u-s^{p}\right) \\
& +c\left(\delta t_{u}-\delta t^{p}\right) \\
& -\delta I_{u}^{p}(t)+\delta T_{u}^{p}(t)+\delta b_{L 1}^{p} \\
& +d_{r e l}^{p}+\Delta p_{a n t} \\
& +\lambda_{1}\left(N_{L 1, u}^{p}+\Delta \phi_{L 1, u}^{p}\right)+\lambda_{1} \varepsilon_{L 1, u}^{p}(t)
\end{aligned}
$$


where,

$$
g_{\hat{u}}^{\hat{p}} \equiv\left[\frac{\partial r_{u}^{p}}{\partial u}\right]_{u=\hat{u}, s^{p}=\hat{s}^{p}}^{\mathrm{T}}=\frac{\left(\hat{u}-\hat{s}^{p}\right)^{\mathrm{T}}}{\left\|\hat{u}-\hat{s}^{p}\right\|} .
$$

The parameters in GR models; (3)-(4) are provided from CLASLIB(SSR2OSR) and MADOCA Products. The satellite position $\left(s^{p}\right)$ and clock errors $\left(\delta t^{p}\right)$ are obtained by CLASLIB(SSR2OSR) or MADOCA Products. Ionosphere delay $\left(\delta I_{u}^{p}\right)$, Tropospheric delay $\left(\delta T_{u}^{p}\right)$, the code bias $\left(\delta b_{C A}^{p}\right)$, the phase bias $\left(\delta b_{L 1}^{p}\right)$ for the satellite $p$, the relativistic delay $\left(d_{r e l}^{p}\right)$, respectively, and the antenna phase center variation (ant, $\Delta p_{a n t}$ ) are obtained from CLASLIB(SSR2OSR). Finally, the receiver position $u$, receiver clock error $\left(\delta t_{u}\right)$ and the interger ambiguity $\left(N_{L 1, u}^{p}\right)$ are estimated by applying the Kalman filters.

Also, we have the following vector regression equation:

$$
y_{t}=H_{t} x_{t}+v_{t}
$$

where,

$$
\begin{gathered}
y_{t} \equiv\left[\begin{array}{c}
\rho_{C A, u}^{p} \\
\Phi_{L 1, u}^{p}
\end{array}\right], \quad H_{t} \equiv\left[\begin{array}{ccc}
\boldsymbol{G}_{\hat{u}}^{\hat{p}} & \mathbf{1} & O \\
\boldsymbol{G}_{\hat{u}}^{\hat{p}} & \mathbf{1} & \lambda_{1} I
\end{array}\right], \\
x_{t} \equiv\left[\begin{array}{c}
u \\
c \delta t_{u} \\
N_{L 1, u}
\end{array}\right], \quad v_{t} \equiv\left[\begin{array}{c}
e_{C A, u}^{p} \\
\varepsilon_{L 1, u}^{p}
\end{array}\right] .
\end{gathered}
$$

where, $\mathbf{1} \equiv[1,1, \cdots, 1]^{\mathrm{T}}: \quad n_{s} \times 1$ vector and $O \equiv$ $[0,0, \cdots, 0]^{\mathrm{T}}: n_{s} \times n_{s}$ matrix. where $n_{s}$ is the number of satellites corresponding to the focusing satellite system. Also define the $n_{s} \times 3$ matrix, and $I$ is the $n_{s} \times n_{s}$ matrix identity as follows:

$$
\boldsymbol{G}_{\hat{u}}^{\hat{\hat{p}}} \equiv\left[\begin{array}{c}
g_{\hat{\hat{u}}}^{\hat{1}} \\
g_{\hat{u}}^{\hat{2}} \\
\vdots \\
g_{\hat{u}}^{\hat{n}_{s}}
\end{array}\right], I \equiv\left[\begin{array}{cccc}
1 & 0 & \ldots & 0 \\
0 & 1 & \ldots & 0 \\
\vdots & \vdots & \ddots & \vdots \\
0 & 0 & \ldots & 1
\end{array}\right]
$$

And then, Kalman filter is applied to the observation equation (5) for GNSS PPP implementation.

\section{QZSS(Quasi-Zenith Satellite System)}

The QZSS (Quasi-Zenith Satellite System) is a regional space-based positioning system that uses a constellation of satellites placed in multiple orbital planes. The system covers regions in East Asia and Oceania centering on Japan and is designed to enable users in the coverage area to receive QZS (Quasi Zenith Satellite) signals from a high elevation angle at all times [6].

\subsection{CLAS}

CLAS use Japan's GNSS-based control stations data to calculate correction information, and utilizing the L6 signal of QZS to broadcast corrections for the Japanease homeland and the surrounding ocean. This technology can be accomplished with centimeter level error.

\subsubsection{CLASLIB(SSR2OSR)}

CLASLIB(CLAS Test Library) is an open source software tool to provide for CLAS. We can get CLASLIB from QZSS website [7]. SSR2OSR is contained in CLASLIB. SSR2OSR is a conversion utility to make offset values of observations from Compact SSR (State Space Representation). The following measurement models is used SSR2OSR. From (3)-(4):

$$
\begin{aligned}
\rho_{C A, u}^{p} \cong & g_{\hat{u}}^{\hat{p}}\left(u-s_{b r d}^{p}\right) \\
& +c\left(\delta t_{u}-\delta t_{b r d}^{p}\right) \\
& +s_{C L A S}^{p}-\delta t_{C L A S}^{p}+\operatorname{PRC} 1+e_{C A, u}^{p}(t), \\
\Phi_{L 1, u}^{p} \cong & g_{\hat{u}}^{\hat{p}}\left(u-s_{b r d}^{p}\right) \\
& +c\left(\delta t_{u}-\delta t_{b r d}^{p}\right) \\
& +s_{C L A S}^{p}-\delta t_{C L A S}^{p}+\mathrm{CPC} 1+\lambda_{1} N_{L 1, u}^{p} \\
& +\lambda_{1} \varepsilon_{L 1, u}^{p}(t) .
\end{aligned}
$$

where,

$$
\begin{aligned}
\mathrm{PRC} 1= & \delta I_{u}^{p}(t)+\delta T_{u}^{p}(t)+\delta b_{C A}^{p} \\
& +d_{r e l}^{p}+\Delta p_{\text {ant }}, \\
\mathrm{CPC} 1= & -\delta I_{u}^{p}(t)+\delta T_{u}^{p}(t)+\delta b_{L 1}^{p} \\
& +d_{r e l}^{p}+\Delta p_{\text {ant }}+\lambda_{1} \Delta \phi_{L 1, u}^{p} .
\end{aligned}
$$

The parameters in SSR2OSR models; (7)-(8) are provided from Broadcast Ephemeris. The satellite position $\left(s_{b r d}^{p}\right)$ and clock errors $\left(\delta t_{b r d}^{p}\right)$ are obtained by Broadcast Ephemeris, Orbit correction $\left(s_{C L A S}^{p}\right)$ and Clock correction $\left(\delta t_{C L A S}^{p}\right)$ are obtained by CLAS. Orbit correction and Clock correction are component in the line of sight from the receiver to the satellite $[\mathrm{m}]$. (PRC1) is pseudo range correction as OSR for CA $[\mathrm{m}]$ and $(\mathrm{CPC} 1)$ is carrier range correction as OSR for $\mathrm{L} 1[\mathrm{~m}]$.

\subsection{MADOCA}

JAXA has developed Multi-GNSS orbit and clock estimator called MADOCA. Estimate satellite orbit and satellite clock from about 100 reference stations that is call MGMnet(Multi GNSS Monitor Network), and multi GNSS support. MADOCA product provide the two method; Real-time or Offline products. We use the Offline products. 


\section{EXPERIMENTAL RESULTS}

We have carried out the experiments of using the GPS data (see Table 1) from the antennas, and the coordinates of their true positions in the WGS-84 system are listed in Table 2 which are obtained by GNSS Earth Observation Network System.

Table 1: Experimental Conditions

\begin{tabular}{|c|c|}
\hline Date & February 5, 2019 \\
\hline Data1(GPST) & $(06: 00$ '20 06:59'59) \\
Data2(GPST) & $(07: 00$ '20 $\sim 07: 59$ '59) \\
Data3(GPST) & $(18: 00$ '20 18:59'59) \\
Data4(GPST) & $(19: 00$ '20 $19: 59$ '59) \\
\hline Location & Tsukuba1, Japan (GEONET) \\
\hline Antenna(Ant) & TPSCR.G5 GSI \\
\hline Receiver & TRIMBLE NETR9 \\
\hline Epoch interval & 1 [s] \\
\hline Elevation angle mask & 15eg.] \\
\hline Measurement Data & C/A code, L1 carrier-phase \\
\hline
\end{tabular}

Table 2: True positions

\begin{tabular}{|c|c|c|}
\hline $\mathrm{X}[\mathrm{m}]$ & $\mathrm{Y}[\mathrm{m}]$ & $\mathrm{Z}[\mathrm{m}]$ \\
\hline-3957162.3949 & 3310203.4909 & 3737752.3012 \\
\hline
\end{tabular}

We have carried out experiments to compare the estimation accuracy of CLAS and MADOCA Products (Table 3).

Table 3: Experimental methods

\begin{tabular}{|c||c|c|}
\hline & MADOCA-PPP & CLAS-PPP \\
\hline \hline Orbit correction & MADOCA(final) & CLAS(ORB) \\
\hline Clock correction & MADOCA(final) & CLAS(CLK) \\
\hline Ionosphere delay & GIM(IGS-final) & CLAS(Iono) \\
\hline Troposphere delay & $\begin{array}{c}\text { Saastamoinen model } \\
\text { + Chao mapping } \\
\text { function }\end{array}$ & CLAS(Trop) \\
\hline
\end{tabular}

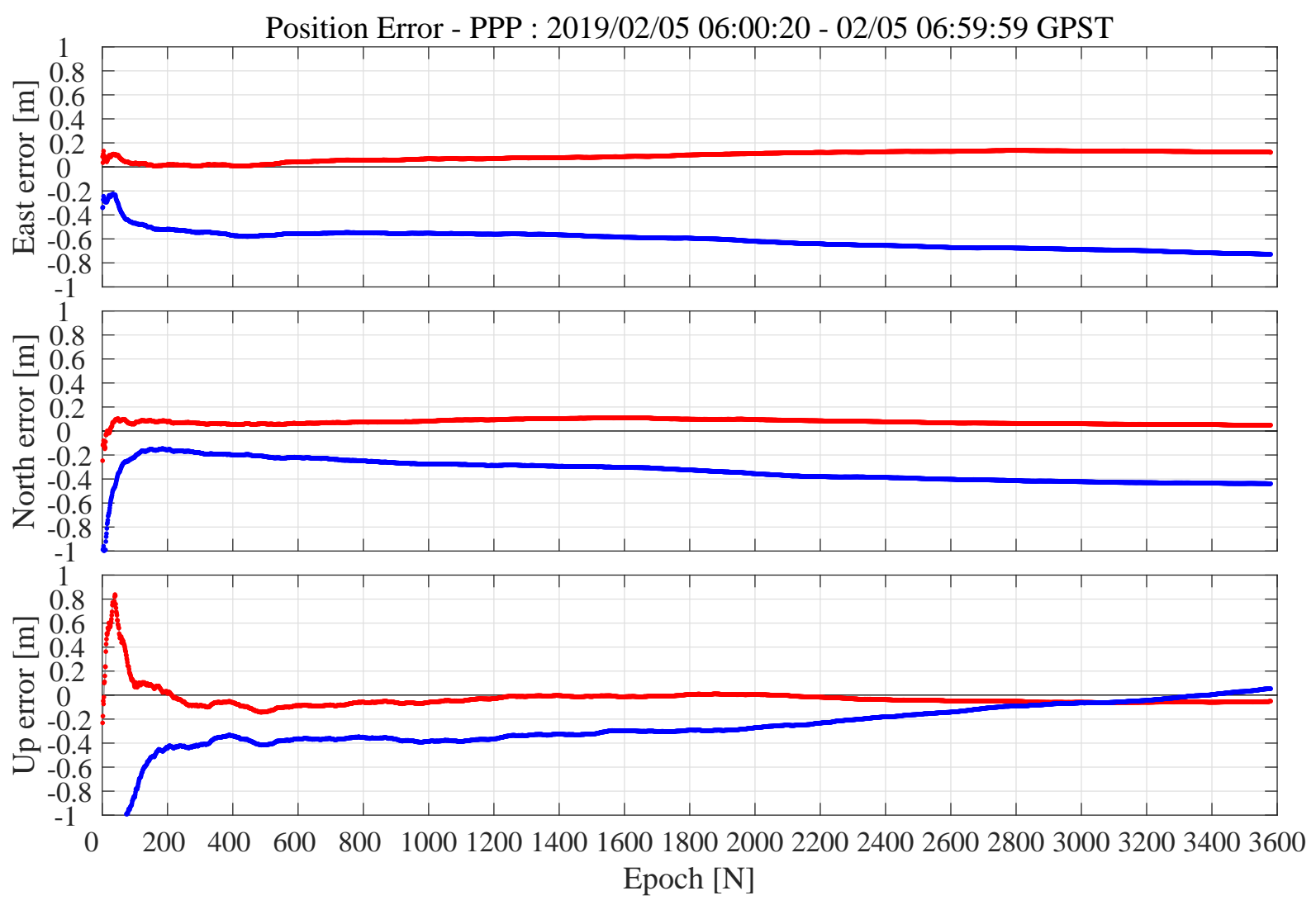

Fig. 1: Positioning Errors at Data1 
Position Error - PPP : 2019/02/05 07:00:20 - 02/05 07:59:59 GPST

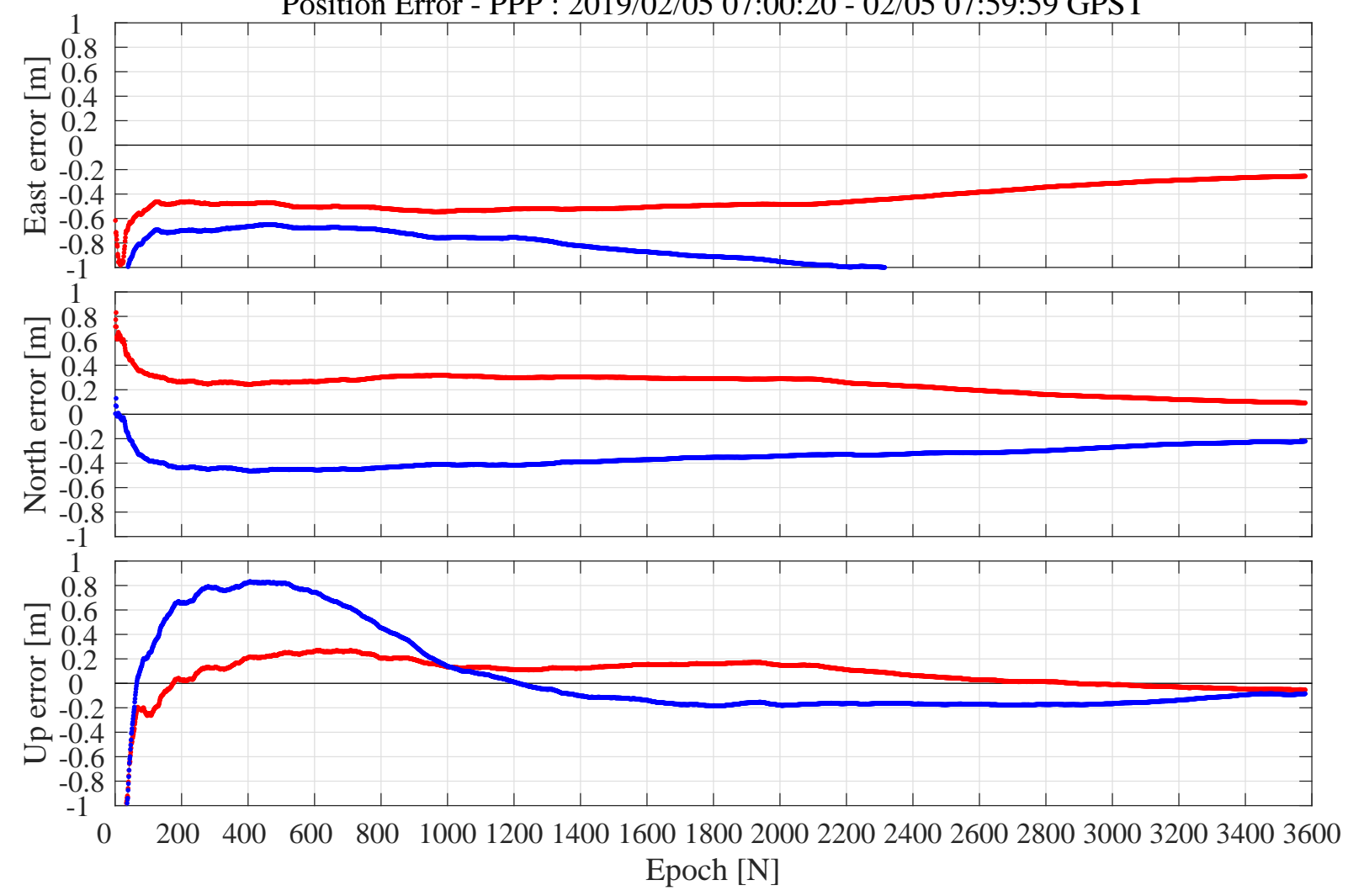

Fig. 2: Positioning Errors at Data2

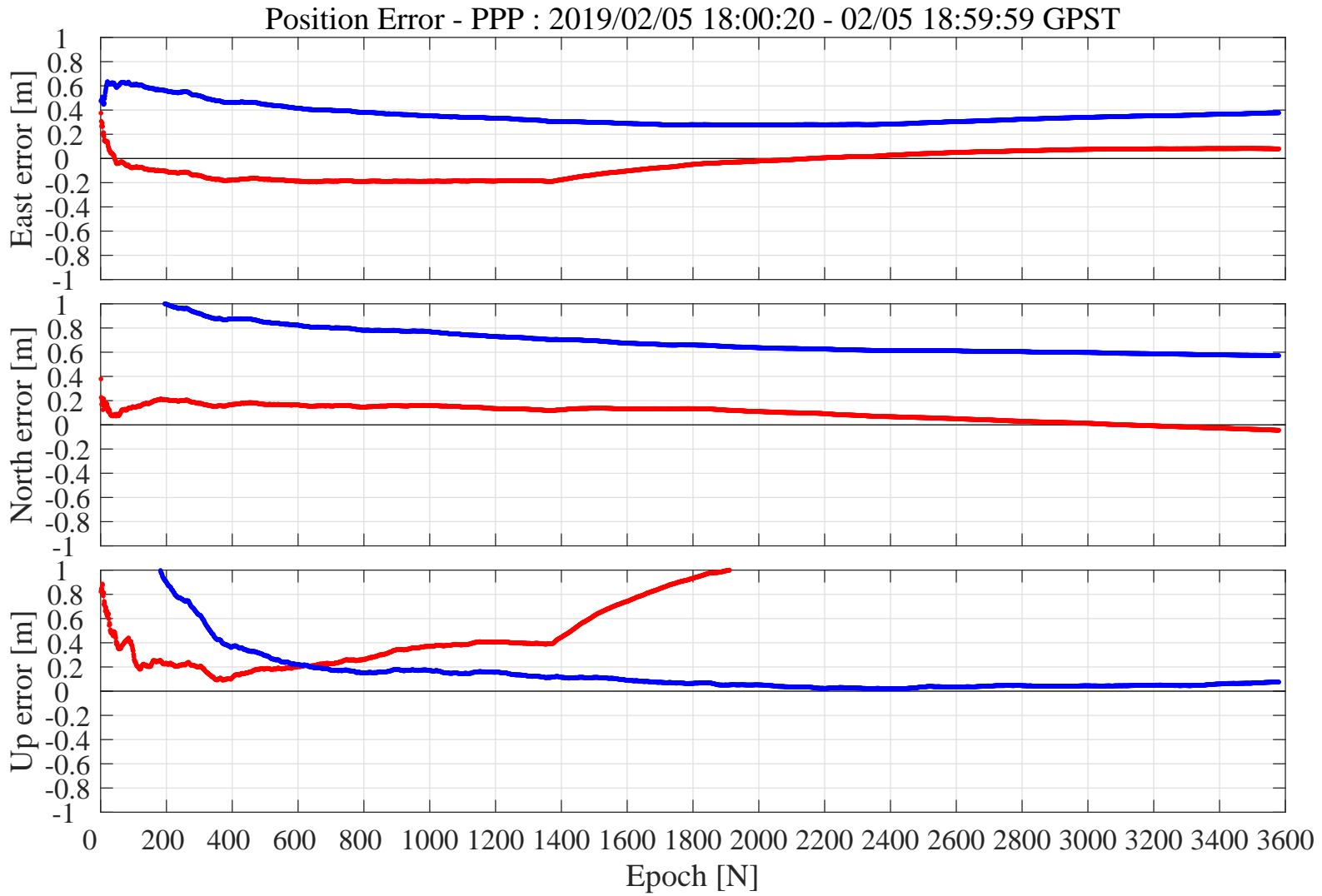

Fig. 3: Positioning Errors at Data3 


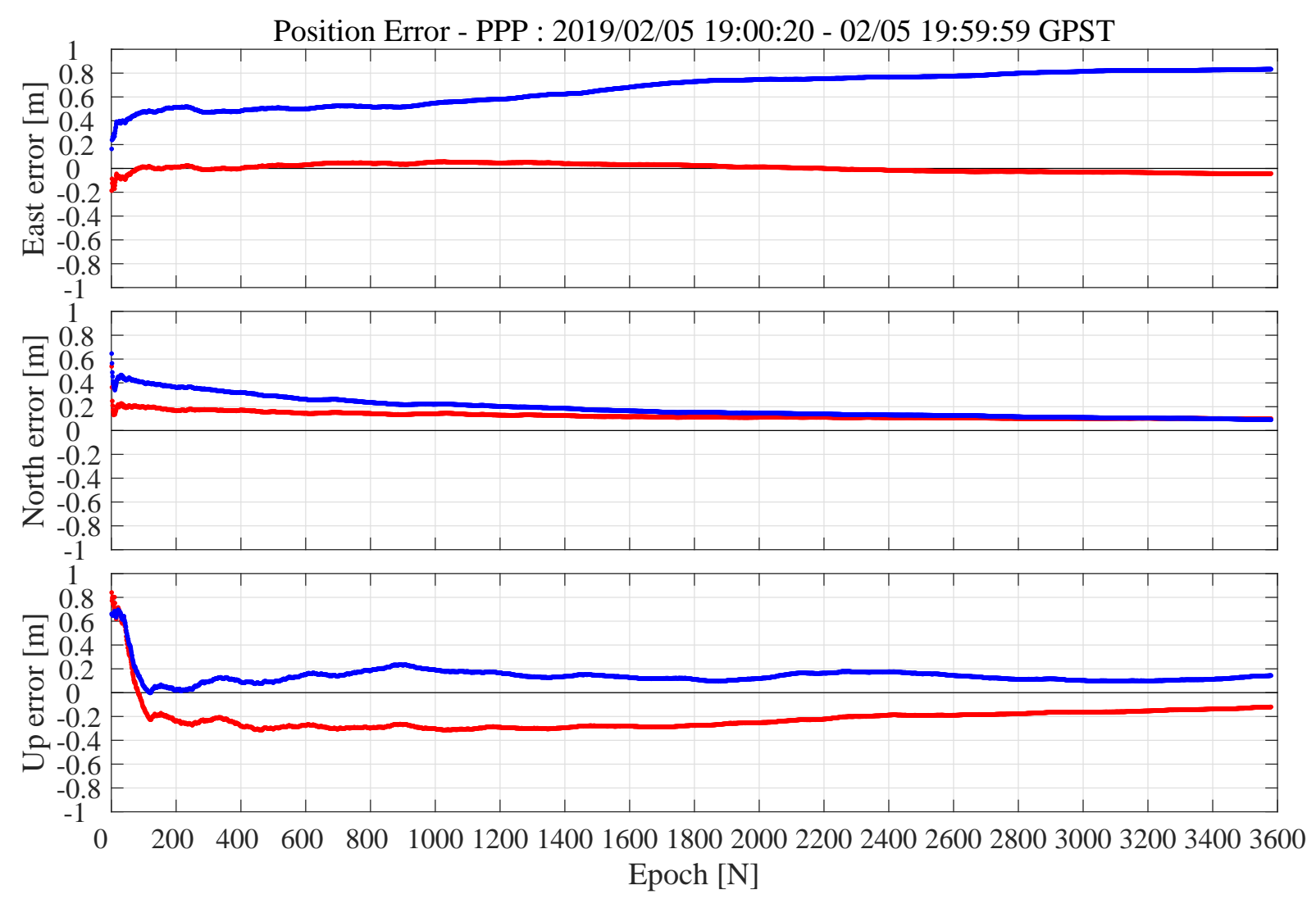

Fig. 4: Positioning Errors at Data4

Table 3 : RMS-ERRORS

\begin{tabular}{|c|c|c|c|c|}
\hline & & & $\mathrm{RM}$ & {$[\mathrm{m}]$} \\
\hline \multirow{6}{*}{ Data1 } & \multirow{3}{*}{ CLAS } & $\mathrm{E}$ & 0.0987 & \multirow{3}{*}{0.0915} \\
\hline & & $\mathrm{N}$ & 0.0790 & \\
\hline & & $\mathrm{U}$ & 0.0957 & \\
\hline & \multirow{3}{*}{ MADOCA Products } & $\mathrm{E}$ & 0.6128 & \multirow{3}{*}{0.4713} \\
\hline & & $\mathrm{N}$ & 0.3455 & \\
\hline & & $\bar{U}$ & 0.4140 & \\
\hline \multirow{6}{*}{ Data2 } & \multirow{3}{*}{ CLAS } & $\mathrm{E}$ & 0.4564 & \multirow{3}{*}{0.3286} \\
\hline & & $\mathrm{N}$ & 0.2581 & \\
\hline & & $\mathrm{U}$ & 0.2214 & \\
\hline & \multirow{3}{*}{ MADOCA Products } & $\mathrm{E}$ & 0.9135 & \multirow{3}{*}{0.6078} \\
\hline & & $\mathrm{N}$ & 0.3577 & \\
\hline & & $\mathrm{U}$ & 0.3821 & \\
\hline \multirow{6}{*}{ Data3 } & \multirow{3}{*}{ CLAS } & $\mathrm{E}$ & 0.1197 & \multirow{3}{*}{0.5181} \\
\hline & & $\mathrm{N}$ & 0.1178 & \\
\hline & & $\bar{U}$ & 0.8816 & \\
\hline & \multirow{3}{*}{ MADOCA Products } & $\mathrm{E}$ & 0.3657 & \multirow{3}{*}{0.5658} \\
\hline & & $\mathrm{N}$ & 0.7214 & \\
\hline & & $\mathrm{U}$ & 0.5535 & \\
\hline \multirow{6}{*}{ Data4 } & \multirow{3}{*}{ CLAS } & $\mathrm{E}$ & 0.0328 & \multirow{3}{*}{0.1628} \\
\hline & & $\mathrm{N}$ & 0.1283 & \\
\hline & & $\mathrm{U}$ & 0.2490 & \\
\hline & \multirow{3}{*}{ MADOCA Products } & $\mathrm{E}$ & 0.6836 & \multirow{3}{*}{0.4221} \\
\hline & & $\mathrm{N}$ & 0.2054 & \\
\hline & & $\mathrm{U}$ & 0.1578 & \\
\hline
\end{tabular}

Figs.1-4 shows the positioning errors with the local level axes (East, North and Upper), where the solid red lines show the errors for using CLAS and the solid blue lines show the errors for using MADOCA Products. The errors were computed by difference between the estimated positions and the coresponding positions in the coordinates of Table 3 . We can observe from Figs.1-4 that the positioning quality is considerably improved by using CLAS. The statistics of the positioning results are summarized in Table 3, where RMS errors are shown for using CLAS and MADOCA Products.

\section{Conclusions}

We have presented PPP algorithms with applying CLAS. When applying CLAS, the positioning accuracy is better than one applying MADOCA Products. Namely, throughout the numerical experiments in the section 5, the total 3DMMS error by applying CLAS is approximately 0.28 [m], in contrary with the total 3DMMS error by applying MADOCA Productsis being aproximately 0.52 [m].

\section{REFERENCES}

[1] N. Motooka, R. Hirokawa, K. Nakakuki, S. Fujita , M. Miya and Y. Sato, Mitsubishi Electric Corpo- 
ration: CLASLIB: An open-source toolkit for lowcost high-precision PPP-RTK positioning Proc. ION GNSS+ 2019, Miami, Florida (Sep. 2019).

[2] Satoshi Kogure: On the developments of MADOCA and MADOCA-PPP, 2016 (in Japanese), http: //www.ic-madoca.org/documents / 2_handout_JAXA.pdf

[3] H. Hasegawa, D. Mimura, Y. Shibayama, Y. Kubo and S. Sugimoto: VPPP algorithms with multiple antennas by applying MADOCA products Proc. of the 50th ISCIE Int. Symp. on Stochastic Systems Theory and Its Applications, Kyoto, Japan (Nov. 2019).

[4] S. Sugimoto and Y. Kubo: GNSS regressive models and precise point positioning, Proc. of the 36th ISCIE
Int. Symp. on Stochastic Systems Theory and Its Applications, pp. 159 - 164, Saitama, Nov. (2004).

[5] S. Sugimoto: Positioning algorithms by GNSS regression models, Section 7.2 in GPS Handbook (in Japanese), pp. 163-185, ed. by S. Sugimoto and R. Shibasaki, Asakura-Shoten, Tokyo, Japan (2010).

[6] National Space Secretariat, Overview of the Quasi-Zenith Satellite System (QZSS): http://qzss.go.jp/en/overview/ services/sv01_what.html

[7] CLAS test library: CLASLIB ver.0.6.0 Manual, February 27, 2019, http://sys.qzss.go.jp/dod/ downloads/download.html?TECH_ID $=36$ 